calculi are not always there. Sometimes a calcified gland casts a shadow which is indistinguishable from that of a calculus. Even when there is a calculus in the ureter do not be in a hurry to operate, because it is probable that sooner or later the calculus will be passed. If you do decide to operate, be sure that the calculus is there at the time of your operation, because very often the mere shock of the patient thinking of the operation is enough to make him pass the stone. You should have a skiagram taken as near to the actual time of the operation as possible, and be sure that all the urine he passes is drained through muslin, to be sure that he does not pass a stone in the meantime. I have known a stone passed between the taking of a skiagram at I I.3O and 2 o'clock, the time fixed for the operation.

And, lastly, one word on saying, "I do not know." There are often occasions in one's life when one cannot make a diagnosis, when one is utterly baffled and does not know what the patient is suffering from. In those cases, I think, the best course is openly to say so. There is nothing to be ashamed of in not being able to tell what is going on inside the patient, and it is better to be open about it and say right out "I do not know." It is related that on one occasion the great Abernethy saw the son of a peer, whose case completely baffled him. In his blunt way he said to the father, "I don't in the least know what is the matter." "Well, Dr. Abernethy," said the peer, "if you don't know, is there anybody who can tell me?", "Yes, sir," replied Abernethy, "plenty, if you go to somebody who is sufficiently ignorant!"

\section{THE USES AND LIMITATIONS OF LIGHT TREATMENT.}

BEING A LECTURE DELIVERED

\author{
$B y$ F. D. HOWITT, \\ M.D., M.R.C.P.
}

AT THE PRINCE OF WALES GENERAL HOSPITAL.

(In connection with the Post-Graduate Course of The Fellowship of Medicine.)

THE first essential to be grasped about light treatment is the fact that it is merely an attempt to supply artificially a natural want. When a physician orders colchicum for gout, or salvarsan for syphilis, he is prescribing a drug which, either as the result of prolonged clinical experience, or of diligent research in the laboratory, has proved itself a valuable specific remedy for the particular disease in which it is employed. But the case of "light treatment" is different. It is not a specific remedy for a particular disease ; still less is it a specific remedy for every disease, as some of its protagonists would have us believe. It is merely a treatment designed to supply a natural want of the living beings.

If an animal is deprived of sunlight, either in quantity or degree, some pathological condition will follow just as surely as if it is deprived of food or water, air or exercise.

These facts were not unknown to the ancients, who appreciated very thoroughly the healing power of the sun, just as they realized it to be the source of life and energy. At Epidauros (the Temple of Esculapius) the outlines of a vast sanatorium, where sun baths, hydrotherapy, massage and other treatments on purely rational lines were carried out, can still be seen. But, like so many rational therapeutic measures, sun treatment seems to have been relegated to the background by the various strange principles which characterized medicine in the Middle Ages, only to be revived in recent times with greater fervour. And so, 
to-day, it is carried out on systematic lines in Switzerland and elsewhere, and is recognized as the treatment of choice for many intractable conditions.

Radiant energy in all its forms is transmitted through the ether at the same speed, viz., I86,000 miles a second, but in varying undulations. The quality of the radiation depends upon the length of the waves, measured from crest to crest. It follows that the shorter the waves the greater is their frequency. The unit of measurement of these waves usually adopted is the Angstrom unit $(\dot{A})$ which is one ten-millionth of a millimetre. From shortest to longest the known wave lengths are as follows :-

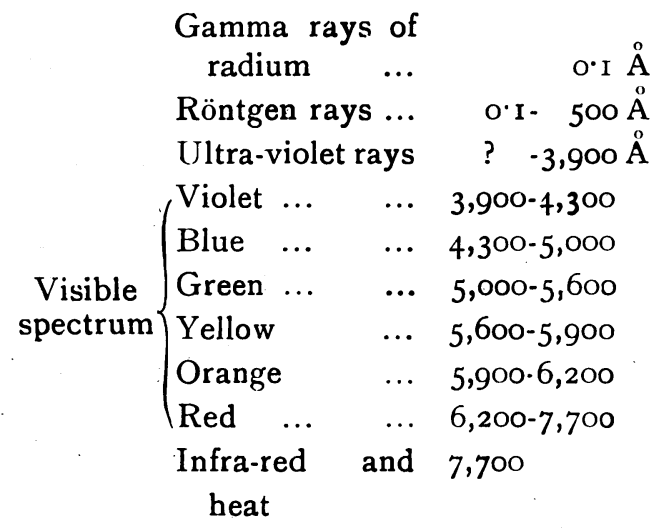

Up to I2) Waves resulting from high-frekilometres quency currents, \&c., and those in length used in wireless telegraphy.

From this it will be seen that the radiations exciting the human retina form a very small portion of the whole.

Artificial light treatment is merely an attempt to emulate the beneficial effects of the sun, and to produce these effects where the natural source is unobtainable.

Finsen, to whom the credit of modern light therapy is chiefly due, working in Denmark, a country where the sun has comparatively little effect, set out to copy and supply this energy artificially, and made the carbon-arc lamp which goes by his name. Since his death, much work has been done in an attempt to discover the physiological and biochemical properties of light and ultra-violet rays, but we still remain in ignorance of most of these.

Now it has been found by experiment that the radiations of shorter wave length, i.e., those in the ultra-violet and violet end of the visible spectrum, have the greatest effect on the skin and the nerves therein. They produce an erythema which reaches a maximum in about six hours. This is followed by desquamation, and, if the exposure has been too severe, by blistering. The erythema is a dilatation of the capillary circulation, caused by the formation of a certain irritative substance in the skin. These rays have also the power of producing in the skin: (a) Chemical effects, of which the activation of cholestrol may be mentioned ; (b) a direct bactericidal action upon microorganisms situated in the skin.

But although these ultra-violet radiations produce the most powerful effects, they are unable to penetrate deeply into the skin, and we find that the shorter the wave length, the less is the penetrative power. Rays in the visible spectrum penetrate more deeply and produce effects in the blood-stream of which the following may be mentioned :

(I) An increase of red cells in cases of anæemia and of hxemoglobin. Normal blood is not affected.

(2) A complicated alteration in the differential white cell count. The effect on the white cells is by no means constant. Usually there is a temporary diminution in the total count, which may be severe in cases of over-dosage, followed by an increase, in which the relative polymorphonuclear count predominates over the lymphocytes.

(3) An increase in the ionic calcium content of the blood-serum. Calcium metabolism has been the subject of much recent research, and it would appear that in many conditions the calcium is not in a free and assimilable form. Light has an action here, possibly in conjunction with its stimulation of the parathyroid glands, and is of value in such conditions as tuberculosis, 
rickets, and certain forms (particularly in children) of urticarial eruptions.

(4) An alteration of the albumin-globulinfibrinogen ratio of the blood-serum. If a small quantity of blood is taken from a normal person, citrated, and left to stand for one hour in a $200 \mathrm{~mm}$. burette, it will be found that the cells have settled so slowly, that a layer of only about $3 \mathrm{~mm}$. of clear plasma remains. In a person whose resistance is destroyed, as for example in phthisis, the cells have dropped so quickly as to leave up to $100 \mathrm{~mm}$. clear plasma. As the patient improves in health, the reading gradually approaches normal, and this test, known as the sedimentation test, is applied in Denmark and elsewhere as an index of the patient's return to health while undergoing light treatment.

(5) An increase in the bactericidal index. This is a most important effect of light treatment. If a given number of colonies of (say) staphylococci are grown on a plate of blood before treatment, and the experiment is repeated after treatment, it is found that the power of the blood to destroy these colonies has been substantially increased.

(6) Stimulation of the sympathetic nervous system and of the ductless glands. It has been shown that the weights of the endocrine glands of rabbits actually undergo a hypertrophy of their parenchymatous tissue as a result of irradiation by light. Reference to the association of calcium metabolism with the parathyroid glands has already been made.

We will not concern ourselves with the effects of the infra-red and heat waves except to note that there is evidence to show that in their presence the effects enumerated above are increased.

The sun, then, produces its different effects upon the animal organism by virtue of these different radiations, and normally our various requirements are supplied by these rays working in conjunction with each other. A deficiency of any particular ray may produce a definite pathological condition. It is obvious, therefore, that in prescribing light treatment rationally for any given condition, we must recognize exactly what rays are required. Roughly, cases fall under two headings :

A. Those requiring chiefly pure ultraviolet radiation. The sources used in artificial phototherapy for this purpose are the mercury-vapour and the tungsten or iron arcs. Of these, the mercuryvapour is much to be preferred as it yields a more even spectrum, it is less messy, it has no fumes, and it is more easily controlled.

B. Those requiring chiefly the more penetrative effect of visible rays. This is produced by the Finsen and other types of the carbon-arc lamp. Pure carbons give a spectrum approximating to that of the sun itself, and therefore, for most purposes, give the best results.

It is necessary in order to deal with different types of case to be in possession of both these sources of radiation. The carbons manufactured with cores of tungsten and other metals are not satisfactory.

Conditions best treated under heading A.(With the mercury vapour lamp).

Chronic Ulcers and Sinuses.-The direct bactericidal action of ultra-violet light on organisms situated in the skin will often heal these conditions where other antiseptics have failed.

Staphylococcal and Streplococcal Infections of the Skin.-CSycosis barbæ, for instance, often yields remarkably to a course of ultra-violet light, and in my opinion it is much better and safer to treat it in this way than by $\mathrm{X}$-rays. In acne vulgaris the best method is to give ultra-violet locally combined with general carbon-arc baths. Strong doses of alkalis should be administered and perhaps sulphur in some form, such as injections of contramine. It is very seldom that acne cannot be made to disappear if this routine is adopted. 
Trophic Diseases of the Skin.-Prurigo, especially of the senile type, often disappears. I have seen alopœcia areata of very long standing disappear after irradiation by ultraviolet light; too often to be attributed to the spontaneity with which this disease is apt to clear up. Sometimes, on the other hand, there is no improvement.

Certain Functional Nerve Cases.-Chronic, rather than acute depressions, and inferiority psychoneuroses derive great benefit. The stimulation of the peripheral nerve-endings and the consequent feeling of well-being are probably responsible. It is well in treating severe cases of this kind to keep them warm during the treatment, and even the psychological effect of an additional red lamp has been advocated.

Rickets and Allied Disorders.-The effect here is chemical, and many complicated factors are involved. Ultra-violet light affects the cholesterol content of the skin and activates the fat-soluble vitamin which is present in cod-liver oil. This effect is not produced if the shorter wave lengths are excluded, as, for instance, by the intervention of a glass screen.

Before leaving the subject of pure ultraviolet light, a word of warning would not be out of place with reference to its use in large doses, particularly in the treatment of local conditions. We are here using powerful rays, approaching $\mathrm{X}$-rays in character. We know of the ultimate dangers of $\mathrm{X}$-rays in the production of telangiectasia and even carcinoma, which often do not show themselves for many years. Light treatment is a new arm of science. It is well to bear in mind the possibility of similar disasters after long periods have elapsed.

\section{The condition best treated under heading $B$.} -(With the carbon-arc lamp).

Chronic Ill-health.-Here light treatment offers probably the best results as a means of restoring health and energy. - But it must not be undertaken without a thorough investigation of the cause, nor must it be regarded as anything but an adjunct to treatment when the cause is ascertained.

Chronic ill-health cannot be rationally treated by the wholesale application of light any more than by the indiscriminate use of iron or arsenic or any of the multitudinous remedies which have served the medical profession from time to time. It may manifest itself in the form of anæmia, lassitude, insomnia, and a whole host of symptoms, but is usually the result of one of two causes:-

(I) Infection Somewhere. - Whether the infection be pyorrhœa, tonsillitis, pyelitis, or some hidden suppuration, it must be diligently searched for, and eradicated finally and completely. It is just as criminal to give light treatment as any other form of treatment without such a thorough investigation, and nothing can bring more discredit on a very valuable therapeutic acquisition than such indiscriminate use. Moreover the value of light treatment is enhanced, not retarded, by the administration of properly given drugs and vaccines and other measures under proper medical supervision.

There is one form of chronic ill-health which very often escapes detection, until such serious results have occurred that surgical intervention or rigorous splinting become necessary, and by that time irrevocable damage is often done. That condition is surgical tuberculosis. The results at Leysin, at Alton and elsewhere have put heliotherapy in the forefront in the treatment of this condition, and artificial sunlight is the best substitute where the natural source is unobtainable.

(2) The other important factor in the causation of chronic ill-health is inattention, voluntary or otherwise, to the common-sense principle of keeping fit. Civilization, whilst giving to mankind all sorts of comforts and luxuries, has carried us far away from the natural foods and the fresh air and exercise 
upon which the animal organism depends, and without which it will surely fail. Sunlight is but one of these essentials, and if we are to obtain the best results we should also insist on proper foods and proper ways of living. It is possible for everyone to undergo a course of physical exercises when the more enjoyable and strenuous games are unobtainable.

In the absence of organic disease, a course of treatment on the above lines will be far more efficacious in restoring health than any medicine taken three times a day. A physician who has the courage to prescribe, and a patient who is sufficiently broadminded to accept such a treatment will be rewarded.

Dosage.-The question of dosage will depend upon the following factors :-

(I) The type of radiation, and the strength of current employed.

(2) The distance of the patient from the source.

(3) The disease to be treated.

(4) The general condition of the patient, and his reaction to treatment.

It is essential, if the best resilts are to be obtained, that the operator should have a sound knowledge of clinical medicine, and of the physical and biochemical properties of light, and the normal reactions of the lamps he uses. All sorts of mechanical devices, such as tintometer scáles and pastil indexes, have been thrown on the market with the object of measuring the dosage, but none are of the slightest value if the clinical condition of the patient is not taken into account.

Nor is the question of pigmentation of material assistance. A good deal of controversy has taken place as to whether pigmenters or non-pigmenters do best. Personally, I think the matter is of little importance, and light treatment should not be pushed to the point of producing pigmentation, at any rate in the early stages. Patients, after a long course of sun or carbon-arc treatment take on a dark brown pigmentation, whilst that resulting from the mercury-vapour is of a more yellowish hue, but in neither case should this be taken as an index of dosage.

It is a more common error to overdose than underdose a patient, particularly in the early stages. Light treatment, like vaccines, produces a "negative phase," and in a patient whose resistance to infection is already low, it is not difficult completely to destroy his protective mechanism by giving too large a dose, and thus aggravate the condition. Having overcome this initial difficulty, the dose can be gradually increased until a maximum is reached, but, here again, one can give too much, particularly in weakly patients.

Finally, there are definite contra-indications to light therapy, of which the following are important :-

(I) Acute Illnesses. - No patient suffering from any acute febrile condition should be given light treatment, otherwise his resistance will be still further lowered, and more harm than good will result.

(2) A Tendency to Homorrhage. - Light treatment increases the clotting time of the blood. Active phthisis with hæmoptysis should not be so treated, as many fatal accidents have occurred in this way. The fact should also be borne in mind in dealing with such conditions as hæmophilia or bleeding from the alimentary tract.

(3) Hypersensitiveness of the Skin.-Apart from the rare condition of hæmatoporphyria congenita, there are certain skin conditions whıch are definitely aggravated by the administration of light.

In the few foregoing remarks, an attempt has been made to put artificial light treatment in its proper perspective, and to show that, used with discrimination, it has a most valuable part to play in the treatment of many conditions. It is not, however, an universal panacea, and the best results will only be obtained when its use is confined 
to those cases where there is a physiological demand for it, and when it is applied with a proper and a conscientious knowledge of the subject.

As instances of a few of the types of case which do well with general light treatment, the following very rough case notes are given :-

(I) Acne Vulgaris.-Girl, aged 2r. Acne for five years. Situation : face, particularly chin, also upper part of back. Been treated with vaccines, manganese and other remedies without effect. Started general light baths, using pure carbons. Given diet sheet restricting carbohydrates and advised to take plenty of vegetables, salad, \&c. Contramine injected once weekly. Light baths increased in length of exposure, and intensity of current. Condition aggravated after three treatments. This died down. After six treatments: Pustules became more superficial and described as " not coming to a head." After twelve treatments (one month): Face practically clear and no more pustules. After fifteen treatments: Discharged free. Seen after nine months: Still clear.

(2) Man, aged 33. Laboratory worker. Complained of lassitude and fatigue. Staphylococcal pustules over whole body, chiefly face, and spread by shaving. Pulse rapid. Blood-pressure low, extremely "toxic." Began treatment with very small doses. Initial treatments produced generalized lymphatic enlargement, and aggravation of general condition. One large boil had to be lanced. Treatment persevered with, but still giving very small doses. Urticaria produced after each treatment. After eight treatments patient began to feel better, lymph glands now normal. After twelve treatments: pustules almost gone, and patient feeling much better. Treatment continued for some weeks to improve general resistance.

(3) Prurigo.-Woman, aged 62. Dry, almost ichthyotic skin, with intense irritation. Marks on legs and sidès from scratching.
Had been to several "spas" and tried all sorts of lotions and ointments without effect. Given a course of light treatment. Irritation ceased, and condition had not returned six months afterwards.

(4) Severe Mental Depression.-Woman, aged 57, had suffered for some years from melancholia, and certain obscure depressions, aggravated by the menopause. Condition had become much worse recently and was now almost suicidal, necessitating nurse in constant attention day and night. Refused food and would not engage even in conversation. Had lost weight considerably and become very exhausted. Treated at home first daily, later three times a week with mercury-vapour lamp. Patient did remarkably well, was able to get up, and finally went away for a holiday. Seen six months later, leading practically normal life.

(5) As an example of chronic ill-health, the case of a man, aged 48 , accountant, may be mentioned. On questioning him, it was found that in addition to living in a stuffy office all day, he would work late at night. He took no exercise at all, and drank a considerable excess of whisky. In addition, his teeth were in a bad condition and he was always constipated. He suffered from insomnia and had developed a suspicious cough. A thorough examination of his chest and sputum, however, gave negative results. It was essential here to attend to his general condition, and light baths were given as an adjunct to treatment. He did very well.

(6) Child, aged 2. Typical case of rickets. Fontanelle open, legs bowed, epiphyseal enlargements, protuberant abdomen, badly nourished. Symptoms of sweating and restlessness. X-ray showed typical changes. Treated for four months with mercuryvapour lamp. Gained 4 lb. Symptoms disappeared. X-ray appearances normal. 